\title{
Functional imaging of hippocampal dysfunction among persons with Alzheimer's disease: a proof-of-concept study
}

This article was published in the following Dove Press journal:

Neuropsychiatric Disease and Treatment

22 November 2010

Number of times this article has been viewed

\section{David B Arciniegas ${ }^{1,2}$ \\ Jason R Tregellas 1,3 \\ Donald C Rojas' \\ Burlleen Hewitt' \\ C Alan Anderson 1,2,4}

'Neurobehavioral Disorders Program, Department of Psychiatry, ${ }^{2}$ Behavioral Neurology Section, University of Colorado Denver, Aurora, CO, USA; ${ }^{3}$ Research Service, ${ }^{4}$ Neurology Service, Denver Veterans Affairs Medical Denver, Denver, CO, USA
Correspondence: David B Arciniegas Neurobehavioral Disorders Program, UCD, I300I East 17th Place,

Campus Box F546, Aurora,

CO 80045, USA

Tel +l 3037244998

Fax +I 3037243594

Email david.arciniegas@ucdenver.edu
Abstract: Cholinergic deficits are an early and functionally significant manifestation of Alzheimer's disease (AD). These deficits contribute to impairment of hippocampally mediated information processing, including declarative memory impairments and abnormal auditory sensory gating. A functional imaging technique that facilitates identification of changes in cholinergically dependent hippocampal information processing would be of considerable use in the study and clinical evaluation of persons with this condition. Techniques that interrogate hippocampal function passively, ie, in a manner requiring no cognitive effort or novel task learning during the neuroimaging procedure, would also be especially useful in this cognitively impaired population. The functional magnetic resonance imaging sensory gating paradigm developed at the University of Colorado, CO, USA, is a functional neuroimaging technique that possesses both of these characteristics. We developed a demonstration project using this paradigm in which we passively interrogated hippocampal function in two subjects with probable AD of mild severity. Imaging data were quick and easy in these subjects and served usefully as an initial demonstration of the feasibility of using this neuroimaging method in this population. Preliminary analyses of the data obtained from these subjects identified abnormal blood oxygen level-dependent responses when compared with four healthy comparators, and the pattern of these responses was consistent with impaired function of the auditory sensory gating network. The strengths and limitations of this neuroimaging paradigm and the additional issues that require investigation in order to continue its development into a research and clinical technique for use in this population are discussed.

Keywords: Alzheimer's disease, hippocampus, acetylcholine, functional magnetic resonance imaging

\section{Introduction}

Early identification of Alzheimer's disease (AD) is needed to facilitate timely treatment delivery and to improve quality of life for affected patients and their families. ${ }^{1}$ Cholinergic deficit is present early in the course of $\mathrm{AD}^{2,3}$ and is a likely contributor to the earliest clinical manifestations of this illness, including declarative memory impairments ${ }^{2,3}$ and disturbances of preattentive information filtering (ie, impaired auditory sensory gating). ${ }^{4,5}$ The latter of these impairments reflects cholinergic deficitrelated abnormal hippocampal physiology, disturbances of which are amenable to identification using the functional magnetic resonance imaging (fMRI) auditory sensory gating paradigm. First described by Tregellas et al, ${ }^{6}$ this brief and behaviorally passive fMRI paradigm interrogates the functional integrity of the cholinergically dependent hippocampal-thalamic-frontal information processing network involved in 
auditory sensory gating. ${ }^{6,7}$ Diminished availability or action of acetylcholine to the hippocampus impairs the ability of this structure to perform its inhibitory (ie, information filtering) function in this network. This cholinergic deficit-induced failure of inhibitory function is the putative neurobiological basis for the increased blood oxygen level-dependent (BOLD) signal (ie, excessive activation) in the hippocampus that is evoked by the auditory sensory gating paradigm. ${ }^{6}$

The fMRI auditory sensory gating paradigm therefore might be a viable method by which to identify AD-induced, cholinergic deficit-mediated, hippocampal dysfunction. The primary aim of this proof-of-concept study was to assess the feasibility of evaluating auditory sensory gating among persons with AD with this fMRI paradigm. A secondary and exploratory aim was a preliminary test of the hypothesis that the fMRI auditory sensory gating paradigm evokes greater activation (ie, identifies failed inhibitory function) of the hippocampus in AD subjects when compared with healthy comparison subjects.

\section{Materials and methods}

This study was approved by the Colorado Multiple Institutional Review Board. Only decisionally capable subjects with AD were eligible for study participation, and informed consent was obtained from all study participants.

\section{Subjects}

Subjects were included if clinical interview, neuropsychiatric examination, and medical records review established a diagnosis of probable AD by the National Institute for Neurological and Communicative Disorders and Stroke-Alzheimer's Disease and Related Disorder Association (NINDS-ADRDA) criteria. ${ }^{8}$ Subjects were assessed cognitively using the Mini-Mental Status Examination (MMSE), ${ }^{9}$ Frontal Assessment Battery (FAB), ${ }^{10}$ and Alzheimer's Disease Assessment Scale-Cognitive Subscale (ADAS-Cog). ${ }^{11}$ MMSE $z$-scores ${ }^{12} \leq-2$ were required for a diagnosis of probable AD. Subjects with focal neurological deficits, parkinsonism, a Hachinski Ischemic Scale ${ }^{13}$ score $>4$, neurodevelopmental, substance use, and psychiatric disorders and those taking cognitively impairing medications were excluded. Data from healthy adults studied previously in the laboratory of one of the investigators (JRT) were used to develop a convenience comparison sample for the MRI analyses.

\section{Magnetic resonance imaging}

Studies were performed with a 3T GE MRI system (GE Healthcare, Milwaukee, WI, USA) using a standard quadrature head coil. Imaging parameters were followed as described by Tregellas et al, ${ }^{6}$ as was administration of the fMRI sensory gating paradigm. Briefly, subjects were administered a hearing test in the scanner to set the task volume at $50 \mathrm{~dB}$ above the hearing threshold. Click stimuli consisted of 'broadband chirps', 10 msec in duration, designed to elicit robust cortical responses. The paradigm used a clustered volume acquisition approach to allow a 6-sec silent period following scanner noise for neuronal inhibitory circuitry to reset and allow auditory stimuli to be presented during silence. During the 12 -sec period between scanner noise, subjects heard $4 \mathrm{sec}$ of 1) silence, 2) one click, or 3) multiple ('repeated') clicks with a 0.5 -sec interclick interval. Auditory stimuli were centered at $8 \mathrm{sec}$ after scanner noise ended. The following volume was acquired 4-6 sec after auditory stimulation to capture peak hemodynamic response. Two stimulus presentations comprised one block. Twenty-eight-sec blocks of 'silence', 'one click', or 'repeated clicks' were presented to the subjects over three runs, totaling $21 \mathrm{~min}$. Five blocks of each stimulus type were randomly distributed throughout each run. Between runs, brief conversations were held with subjects to ensure that they were awake and responsive. The contrast 'repeated clicks-one click' was the measure of auditory sensory gating. This subtraction removes most of the effects of overall auditory responsiveness, which was measured separately in the contrast 'one click-silence.' Multiple clicks, rather than a click pair, were used to maximize sensitivity to the difference in BOLD response between the two stimulus conditions.

The fMRI analyses were performed using Statistical Parametric Mapping software version 5 (SPM5; Wellcome Trust Centre for Neuroimaging, London, UK; http://www.fil.ion. ucl.ac.uk/spm/software/spm5/) and used a region of interest (ROI) approach. Data from each subject were realigned to the first volume, normalized to the Montreal Neurological Institute gray-matter template, using a gray-matter-segmented IREPI as an intermediate to improve registration, and smoothed with 8-mm FWHM Gaussian kernel. Data were modeled with an HRF-convolved boxcar function, using the general linear model in SPM5. A 128-sec high-pass filter was applied to remove low-frequency fluctuation in the BOLD signal. A random effects analysis was used to evaluate hippocampal response. The ROI was an $8-\mathrm{mm}$ sphere centered at $x=-24$, $y=-18$, and $z=-15$, the hippocampal location that showed maximal group differences in a previous study using the sensory gating task. ${ }^{6}$ Data within the ROI were small volume corrected at a family-wise error rate of $P<0.05$.

\section{Results}

Two individuals satisfying NINDS-ADRDA criteria for AD were imaged using the fMRI auditory sensory gating 
paradigm. The first was a 71-year-old, right-handed man with a 6-year history of probable AD who was on stable treatment with donepezil $10 \mathrm{mg}$ daily and memantine $10 \mathrm{mg}$ twice daily. His MMSE was $24 / 30(z=-2.5)$, FAB was $11 / 18$, and ADAS$\mathrm{Cog}$ was $22 / 70$. The second was a 78 -year-old, right-handed woman with a 7 -year history of probable AD who was on stable treatment with donepezil $10 \mathrm{mg}$ daily and memantine $5 \mathrm{mg}$ twice daily. Her MMSE was 22/30 ( $z=-3.8)$, FAB was $11 / 18$, and ADAS-Cog was 34/70. Clinical examination and assessment of functional status (obtained by caregiver interview) classified $\mathrm{AD}$ severity in both subjects as mild. Both subjects tolerated the imaging procedure easily, remained alert (per visual monitoring of subjects during scanning by our research assistant), and yielded fMRI data of quality sufficient to subject those data to analyses.

The convenience comparison sample consisted of four healthy subjects drawn from prior studies in our laboratory. The mean age of this group was $52.0 \pm 2.2$ years. As the mean age of the AD group was significantly older (74.5 \pm 4.9 ; $P<0.001$ ), age was used as a covariate in the fMRI data analyses.

Consistent with our a priori hypothesis, subjects with $\mathrm{AD}$ demonstrated abnormal activation of the hippocampus $(t=3.61$; see Figure 1). Single-subject BOLD responses at the hippocampal local maxima, expressed as percent signal change relative to the global mean, are presented in Figure 2.

\section{Discussion}

The fMRI auditory sensory gating paradigm appears to be a feasible method of evaluating the preattentive information-filtering function of the cholinergically dependent hippocampally mediated auditory sensory gating network among persons with AD. In respect of its potential practicality, the fMRI auditory sensory gating paradigm assesses hippocampal function in a single, relatively brief, MR imaging session; such brevity is an important feature for the practical application of fMRI assessments in the AD population. Additionally, it assesses hippocampal function using a passive protocol; the only in-scanner behavioral requirement of persons with $\mathrm{AD}$ is to remain awake. As dementia due to $\mathrm{AD}$ entails difficulty learning new information and executing complex tasks, imaging paradigms that interrogate hippocampal function by passive means, like ours and that of others, ${ }^{14}$ offer potential practical advantages over those that require subjects to learn and perform novel inscanner cognitive tasks. ${ }^{15-18}$ The unimodal imaging approach employed here also offers potential feasibility advantages over others that, although robust, may be relatively more time and resource intensive in respect of data acquisition and analyses. ${ }^{14,19,20}$ Additionally, the emphasis on hippocampal function rather than structure is an important feature of this approach, given the variable relationship between hippocampal structure and clinical symptoms in the early stages of AD. ${ }^{3,21-24}$

The preliminary analysis of the fMRI data obtained during this demonstration project identified greater BOLD signal in the hippocampus of the two AD subjects when compared with healthy comparison subjects, even after correcting for the between-group age difference. This observation is consistent with our predicted effects of AD neuropathology on the neurobiology of auditory sensory gating. The single-subject BOLD responses at the
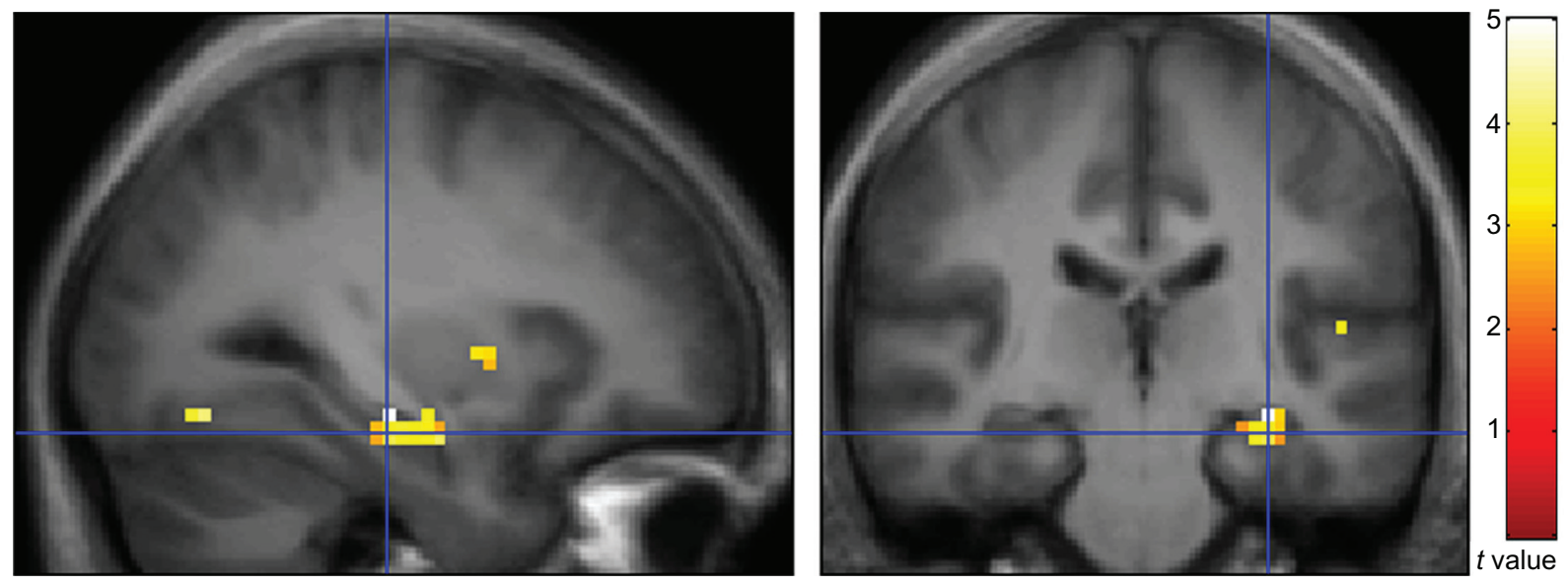

Figure I Sagittal (left) and coronal (right) views of BOLD response during the fMRI auditory sensory gating paradigm (contrast: AD subjects $>$ healthy comparison subjects). These responses are overlaid on an average anatomical image constructed using data acquired from healthy comparison subjects and employ radiological convention (right on left).

Abbreviations: BOLD, blood oxygen level-dependent; fMRI, functional magnetic resonance imaging; AD, Alzheimer's disease. 


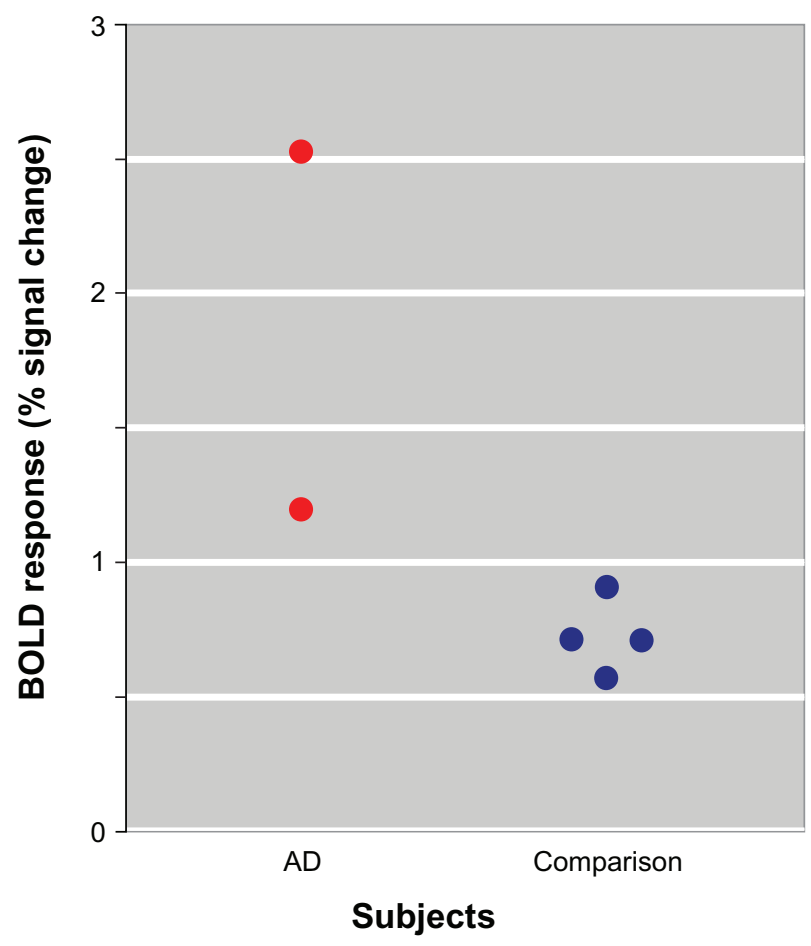

Figure 2 Scatter plot of BOLD responses (\% signal change in the 'repeated clicks-one click' contrast) among subjects with $A D$ and comparison subjects. Each point represents single-subject BOLD responses at the hippocampal local maxima identified in the between-group comparison.

Abbreviations: BOLD, blood oxygen level-dependent; AD, Alzheimer's disease.

hippocampal local maxima identified in the between-group comparison presented in Figure 2 are also noteworthy in that there is no overlap between the AD and healthy comparison subjects. As a proof-of-concept exercise, these data are encouraging of the possibility that, with further development, BOLD responses during the fMRI auditory sensory gating paradigm might contribute usefully to the research and/or clinical assessments of persons with latelife cognitive impairments.

The preliminary nature of this study, however, necessitates cautious interpretation of these findings and suggestions. Replication of this work in a larger sample of subjects with $\mathrm{AD}$ and age- and gender-matched healthy comparison subjects is required to evaluate the usefulness of this fMRI protocol as a method of identifying AD-induced hippocampal dysfunction. Given the preponderance of women affected by this condition and previously observed sexual dimorphism in cerebral responses to fMRI-related auditory stressors, ${ }^{18}$ the possibility of sexual dimorphism in BOLD responses in the fMRI auditory sensory gating paradigm requires consideration and investigation. Additionally, studies are needed to determine whether this paradigm distinguishes individuals with $\mathrm{AD}$ from individuals with memory impairment due to other common late-life neuropsychiatric conditions, especially dementias caused by other forms of neurodegeneration and/or cerebrovascular disease.

It is also important to note that sensory gating impairments are not specific to $\mathrm{AD}$ but are instead features of a wide range of neuropsychiatric conditions, most notably schizophrenia ${ }^{6,25}$ and traumatic brain injury. ${ }^{7}$ A common theme across these conditions is cholinergic dysfunction. Loss of ventral forebrain neurons and their cortical efferents in $\mathrm{AD}^{2,3}$ and traumatic brain injury ${ }^{26-28}$ and (maladaptive) mutation of alpha-7 nicotinic cholinergic receptors in schizophrenia ${ }^{25}$ are the putative bases for auditory sensory gating impairments in these conditions. It is possible that the fMRI auditory sensory gating paradigm will be understood most usefully as an assessment of the integrity of this system independent of clinical diagnosis. If so, then it may not be a marker of early AD per se but instead a marker of any condition in which cholinergic dysfunction occurs, whether by loss of cholinergic neurons, injury to their axons, or altered responsiveness to acetylcholine at their cortical targets. Increased BOLD responses observed in older patients during the fMRI auditory sensory gating paradigm might only narrow the range of potential etiologic explanations for cognitive complaints to those conditions in which cholinergic dysfunction figures prominently (ie, $\mathrm{AD}$, amnestic mild cognitive impairment, diffuse Lewy body disease, traumatic brain injury, and, possibly, cerebrovascular disease).$^{29}$ If such studies demonstrate that this method is useful for any of these purposes at the group and single-patient levels, then the timing and economic implications of its introduction into the diagnostic evaluation of persons with suspected $\mathrm{AD}$ will also need to be considered.

For the present, this initial 'proof-of-concept' demonstration is encouraging of the potential usefulness of the fMRI auditory sensory gating paradigm in $\mathrm{AD}$ research. The present findings suggest that further development and investigation of this neuroimaging technique are warranted.

\section{Disclosure}

The authors report no conflicts of interest in this work.

\section{References}

1. Small GW. Differential diagnosis and early detection of dementia. Am J Geriatr Psychiatry. 1998;6(2 Suppl 1):S26-S33.

2. Mesulam M. The cholinergic lesion of Alzheimer's disease: pivotal factor or side show? Learn Mem. 2004;11(1):43-49.

3. Mesulam M, Shaw P, Mash D, Weintraub S. Cholinergic nucleus basalis tauopathy emerges early in the aging-MCI-AD continuum. Ann Neurol. 2004;55(6):815-828.

4. Boutros N, Torello MW, Burns EM, Wu SS, Nasrallah HA. Evoked potentials in subjects at risk for Alzheimer's disease. Psychiatry Res. 1995;57(1):57-63. 
5. Fein G, Biggins C, van Dyke C. The auditory P50 response is normal in Alzheimer's disease when measured via a paired click paradigm. Electroencephalogr Clin Neurophysiol. 1994;92(6):536-545.

6. Tregellas JR, Davalos DB, Rojas DC, et al. Increased hemodynamic response in the hippocampus, thalamus and prefrontal cortex during abnormal sensory gating in schizophrenia. Schizophr Res. 2007; 92(1-3):262-272.

7. Arciniegas DB, Topkoff JL. Applications of the P50 evoked response to the evaluation of cognitive impairments after traumatic brain injury. Phys Med Rehabil Clin N Am. 2004;15(1):177-203, viii.

8. McKhann G, Drachman D, Folstein M, Katzman R, Price D, Stadlan EM. Clinical diagnosis of Alzheimer's disease: report of the NINCDSADRDA Work Group under the auspices of Department of Health and Human Services Task Force on Alzheimer's Disease. Neurology. 1984; 34(7):939-944.

9. Folstein MF, Folstein SE, McHugh PR. "Mini-mental state". A practical method for grading the cognitive state of patients for the clinician. J Psychiatr Res. 1975;12(3):189-198.

10. Dubois B, Slachevsky A, Litvan I, Pillon B. The FAB: a Frontal Assessment Battery at bedside. Neurology. 2000;55(11):1621-1626.

11. Rosen WG, Mohs RC, Davis KL. A new rating scale for Alzheimer's disease. Am J Psychiatry. 1984;141(11):1356-1364.

12. Crum RM, Anthony JC, Bassett SS, Folstein MF. Population-based norms for the Mini-Mental State Examination by age and educational level. JAMA. 1993;269(18):2386-2391.

13. Hachinski VC, Iliff LD, Zilhka E, et al. Cerebral blood flow in dementia. Arch Neurol. 1975;32(9):632-637.

14. Li SJ, Li Z, Wu G, Zhang MJ, Franczak M, Antuono PG. Alzheimer Disease: evaluation of a functional MR imaging index as a marker. Radiology. 2002;225(1):253-259.

15. Pihlajamäki M, DePeau KM, Blacker D, Sperling RA. Impaired medial temporal repetition suppression is related to failure of parietal deactivation in Alzheimer disease. Am J Geriatr Psychiatry. 2008 16(4):283-292.

16. Diamond EL, Miller S, Dickerson BC, et al. Relationship of fMRI activation to clinical trial memory measures in Alzheimer disease. Neurology. 2007;69(13):1331-1341.

17. Fleisher AS, Houston WS, Eyler LT, et al. Identification of Alzheimer disease risk by functional magnetic resonance imaging. Arch Neurol. 2005;62(12):1881-1888.
18. Tomasi D, Chang L, Caparelli EC, Ernst T. Sex differences in sensory gating of the thalamus during auditory interference of visual attention tasks. Neuroscience. 2008;151(4):1006-1015.

19. Besga A, Ortiz L, Fernandez A, et al. Structural and functional patterns in healthy aging, mild cognitive impairment, and Alzheimer disease. Alzheimer Dis Assoc Disord. 2010;24(1):1-10.

20. Fernandez A, Arrazola J, Maestu F, et al. Correlations of hippocampal atrophy and focal low-frequency magnetic activity in Alzheimer disease: volumetric MR imaging-magnetoencephalographic study. AJNR Am J Neuroradiol. 2003;24(3):481-487.

21. Ridha BH, Anderson VM, Barnes J, et al. Volumetric MRI and cognitive measures in Alzheimer disease: comparison of markers of progression. J Neurol. 2008;255(4):567-574.

22. Fennema-Notestine C, Hagler DJ Jr, McEvoy LK, et al. Structural MRI biomarkers for preclinical and mild Alzheimer's disease. Hum Brain Mapp. 2009;30(10):3238-3253.

23. Karow DS, McEvoy LK, Fennema-Notestine C, et al. Relative capability of MR imaging and FDG PET to depict changes associated with prodromal and early Alzheimer disease. Radiology. 2010;256(3): 932-942.

24. McEvoy LK, Fennema-Notestine C, Roddey JC, et al. Alzheimer disease: quantitative structural neuroimaging for detection and prediction of clinical and structural changes in mild cognitive impairment. Radiology. 2009;251(1):195-205.

25. Freedman R, Adams CE, Adler LE, et al. Inhibitory neurophysiological deficit as a phenotype for genetic investigation of schizophrenia. Am J Med Genet. 2000;97(1):58-64.

26. Murdoch I, Nicoll JA, Graham DI, Dewar D. Nucleus basalis of Meynert pathology in the human brain after fatal head injury. $J$ Neurotrauma. 2002;19(2):279-284.

27. Murdoch I, Perry EK, Court JA, Graham DI, Dewar D. Cortical cholinergic dysfunction after human head injury. J Neurotrauma. 1998; 15(5):295-305.

28. Dewar D, Graham DI. Depletion of choline acetyltransferase activity but preservation of M1 and M2 muscarinic receptor binding sites in temporal cortex following head injury: a preliminary human postmortem study. J Neurotrauma. 1996;13(4):181-187.

29. Schliebs R, Arendt T. The significance of the cholinergic system in the brain during aging and in Alzheimer's disease. J Neural Transm. 2006; 113(11):1625-1644.
Neuropsychiatric Disease and Treatment

\section{Publish your work in this journal}

Neuropsychiatric Disease and Treatment is an international, peerreviewed journal of clinical therapeutics and pharmacology focusing on concise rapid reporting of clinical or pre-clinical studies on a range of neuropsychiatric and neurological disorders. This journal is indexed on PubMed Central, the 'PsycINFO' database and CAS, and is the official

\section{Dovepress}

journal of The International Neuropsychiatric Association (INA). The manuscript management system is completely online and includes a very quick and fair peer-review system, which is all easy to use. Visit http://www.dovepress.com/testimonials.php to read real quotes from published authors. 